\title{
Method inventory for assessment of physical activity at VDU workplaces
}

\author{
Rolf Ellegast, Britta Weber and Rena Mahlberg \\ Institute for Occupational Safety and Health of the German Social Accident Insurance (IFA) \\ Division Ergonomics - Physical Environmental Factors, Alte Heerstraße 111, 53757 Sankt Augustin, Germany
}

\begin{abstract}
Physical inactivity and prolonged static work tasks may seriously affect health. There are numerous indications that promoting physical activity (PA) at sedentary workplaces can reduce these health risks. However, PA interventions have so far been documented rarely on the basis of medical parameters. Effects on the PA behavior are often studied only through the methods of subjective self-assessment. For this reason an extensive method inventory was developed consisting of objective PA assessment methods and various methods for documenting PA related health outcomes. The developed method inventory has been tested in a pilot intervention study at office workplaces. The current paper presents and discusses a part of the applied inventory. The methods considered here demonstrated several positive intervention effects: intervention subjects were more active, felt better, increased muscle strength and showed improvements in resting heart rate and BMI. Not all data has been analyzed to date, but the preliminary results suggest that most of the investigated methods turned out to be suitable for the documentation of intervention effects. Among the methods for which no effects were found, the question remains whether this is due to a lack of sensitivity of the method or due to aspects related to the study design.
\end{abstract}

Keywords: VDU workplaces, intervention study, measurement, physical activity, health outcomes

\section{Introduction}

Today more than $40 \%$ of all employees in the EU are working at visual display units (VDUs) [6]. Several studies have discussed general associations between sedentary work and musculoskeletal disorders [1]. Physical inactivity and prolonged static work tasks may also seriously affect the cardiovascular system [7]. These health risks can be reduced by preventive strategies which promote physical activity (PA) at sedentary workplaces. Accurate and reliable measurement of PA behavior and its attributed health outcomes is considered an essential component of health promotion research and evaluation practice [2] Past effect analysis often rely exclusively on selfreports. To determine effects on the movement behavior, hardly any objective measurement methods were used $[3,4,8]$. Furthermore, intervention effects were rarely quantified on the basis of medical and physiological parameters and the application of medical check-ups [8].

The aim of this study was to develop and test a comprehensive assessment inventory for PA and re- lated health outcomes within a pilot intervention study at office VDU workplaces. In this article results of the evaluation of parts of the assessment inventory for PA will be presented.

\section{Methods}

\subsection{Subjects and experimental design}

25 experienced office workers ( 6 women, 19 men) volunteered as subjects in a randomized controlled trial at VDU workplaces. The intervention group (IG) $(n=13)$ was introduced to a wide-ranging package of PA promoting measures, whereas the control group (CG) $(n=12)$ continued their usual office work. The package consisted of measures aiming at the working conditions (e. g. sit-stand tables) and the behavior (e. g. pedometers as activity feedback, face-to-face motivation for lunch walks etc., an incentive system for bicycle commuting or sports activities). The intervention lasted 12 weeks. 


\subsection{Assessment of physical activity}

During the intervention phase, daily occupational PA was assessed by activity logs and a simple activity measurement system (AiperMotion 320). The activity logs provided the daily time spent sitting, standing and walking whereas the AiperMotion gave information on the amount of time spent actively and number of steps.

Precise assessments before and during the intervention were conducted with an expert measurement system (CUELA Activity System) [11, 12]. This person-centered measuring system consists of seven inertial motion sensors (3D accelerometers and gyroscopes) as well as a miniature data storage unit with a flash memory card, which can be attached to the subject. The sensors were positioned at the thoracic spine (Th3), lumbar spine (L5/S1), the upper arm of the dominant arm, the thighs and lower legs. From the measured signals (sampling rate: $50 \mathrm{~Hz}$ ) body and joint angles and physical activity intensities (PAI) are calculated. PAI values are determined by calculating a sliding root mean square of the highpass filtered vector magnitude of the $3 \mathrm{D}$ acceleration signals (time window: $3 \mathrm{~s}$ ). The CUELA software automatically identifies various activities and body postures and determines energy expenditure [12]. The measurement data can be depicted with the software together with the digitalized video recording of the workplace situation and a $3 \mathrm{D}$ animated figure.

\subsection{Assessment of well-being and medical check-up}

Before and after the intervention several methods were applied to analyze the health outcomes:

A multidimensional mood state questionnaire (MDBF, Mehrdimensionaler Befindlichkeitsfragebogen) was applied to assess the emotional well-being [10]. The MDBF consists of 24 items (each with five-step rating scale) for measuring three bipolar dimensions (scales) of the current mental state: good mood-bad mood, awake-tired, calm-nervous. For analysis, mean values and standard deviations of the scales are calculated and compared with the values of the norm sample. For this purpose, the scores are converted in percentile ranks developed on the basis of the norm sample. A percentile rank value of 60 means for example that $60 \%$ of the norm sample has worse values for the corresponding scale.

A general medical examination (pulmonary and cardiac auscultation, body anthropometrics, vital parameters, reflexes) and the standardized orthopaedic medical check-up G 46 [9] were conducted. This included the identification of joint flexibility and various functionality tests (e.g. finger ground test). The standard population is used to classify the checkup results.

Maximum strength of the back muscles (trunk flexion and extension muscles) was measured using an isometric force meter (Dr. WOLFF's Back-check). The associated software interprets the obtained force values depending on age, height, weight and gender.

Strength endurance of the back, abdominal, shoulder and thigh muscles was determined by standardized tests from the rehabilitation medicine [5]. At this, the subject adopts a standardized posture and remains in this position as long as possible. The achieved time is recorded in seconds.

\subsection{Data processing and statistical analysis}

Two-way ANOVAs were performed for the group comparisons of the pre-post differences. Day-to-day data (activity logs and AiperMotion) were analyzed by Wilcoxon signed-rank tests.

\section{Results}

\subsection{Assessment of physical activity}

The PA assessment revealed several significant positive intervention effects: The activity logs showed that the intervention subjects spent more time standing and less time sitting during the whole period ( $p \leq 0.001)$ compared to the control subjects (see Figures 1 and 2).

The intervention group reported on average additional standing times of $64.4 \pm 18.7 \mathrm{~min}$ in comparison to the control subjects. The daily time spent in sitting postures was correspondingly reduced for the intervention subjects (mean difference 58.3 \pm 19.3 min) in comparison to the control group.

The simple activity measurement system found higher step numbers for the intervention group $(p \leq 0.001)$ (see Figure 3). The dashed line represents the target of $588 \mathrm{steps} / \mathrm{h}$. It results from an $8.5 \mathrm{~h}$ lasting work day, in which ideally 5000 steps should be made. The intervention group reached this target in most cases, whereas the control group missed it most times (see Figure 3).

The expert CUELA measurements revealed significant differences in PA behavior change: A reduction of sitting $(p \leq 0.001)$ and an increase of standing 
$(p \leq 0.001)$ and walking $(p \leq 0.01)$ as well as increased PAI levels of the upper and lower extremities and the trunk (each $\mathrm{p} \leq 0.001$ ) were measured for the intervention group, whereas no significant effects were found for the control group (see Figures 4 and 5 ). For the energy expenditure, no significant group differences were found (see Figure 6).

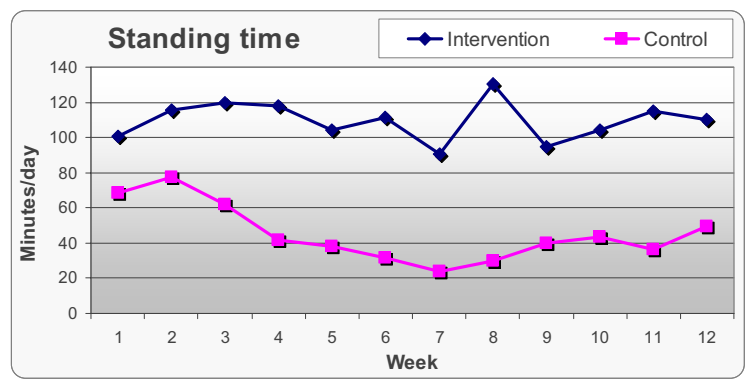

Fig. 1. Daily standing time provided by activity logs (group means per week)

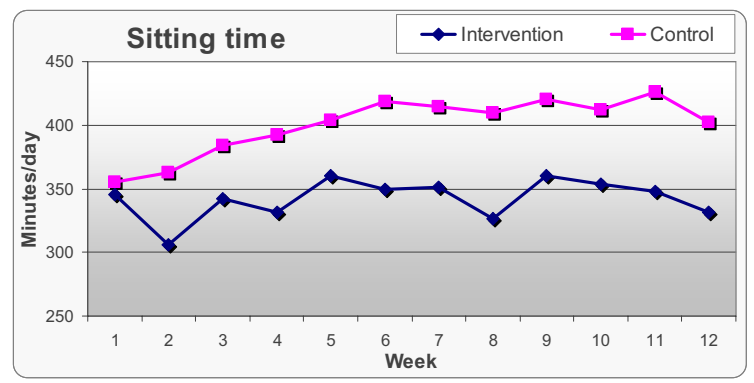

Fig. 2. Daily sitting time provided by activity logs (group means per week)

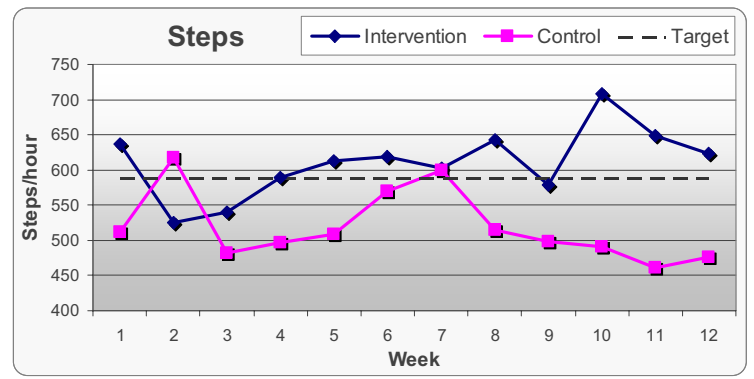

Fig. 3. Steps per hour measured by AiperMotion (group means per week)

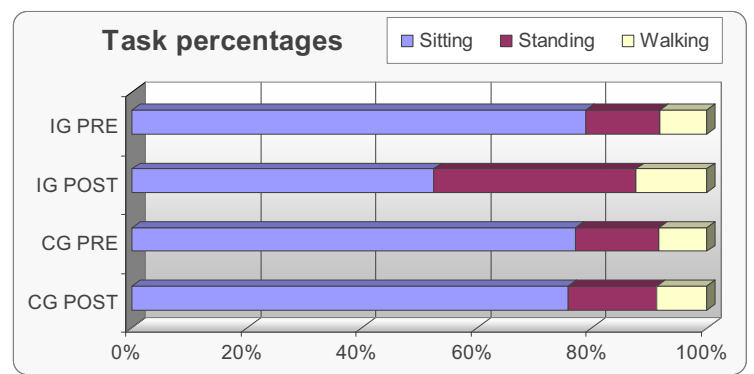

Fig. 4. Pre and post task percentages measured by CUELA (mean per group $(\mathrm{IG}=$ intervention group, $\mathrm{CG}=$ control group $))$.

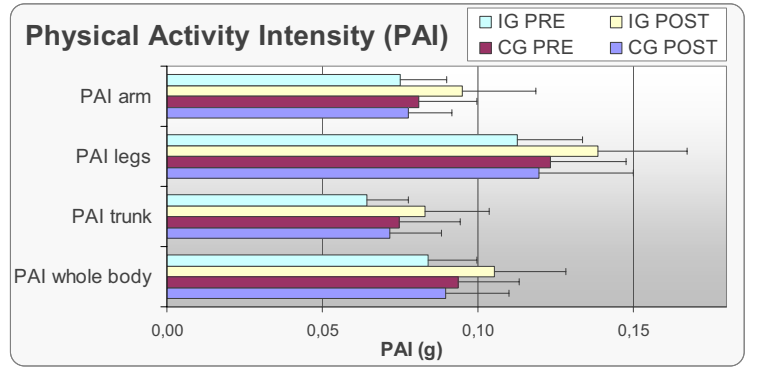

Fig. 5. Pre and post levels of PAI of different body regions measured by CUELA (mean per group ( $\mathrm{IG}=$ intervention group, $\mathrm{CG}=$ control group)).

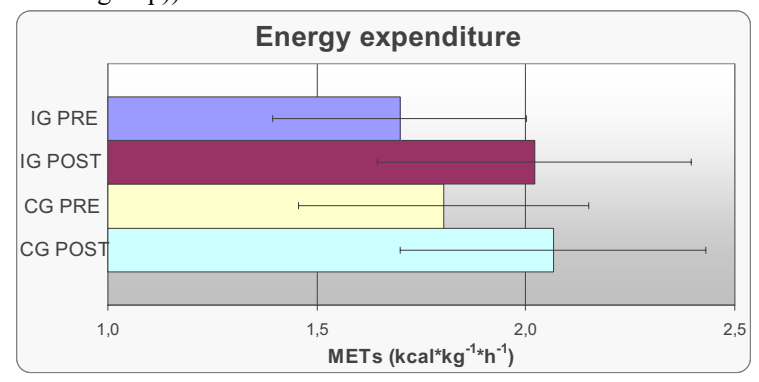

Fig. 6. Pre and post energy expenditure measured by CUELA (mean per group $(\mathrm{IG}=$ intervention group, $\mathrm{CG}=$ control group)).

\subsection{Assessment of well-being and medical check-up}

The applied methods showed some significant positive intervention effects regarding the mental state and the medical parameters:

Intervention subjects showed improvements in body weight/BMI and resting heart rate. The mean BMI of the intervention group was reduced from 26.3 $\pm 3.2 \mathrm{~kg} / \mathrm{m}^{2}$ to $25.6 \pm 3.1 \mathrm{~kg} / \mathrm{m}^{2}$ for the pre-post comparison. After intervention the resting heart rate slightly decreased for the intervention group and increased for the control group (see Table 1).

Generally the MDBF assessment led to better rank values (> 50 percentile rank) for the intervention and control group compared to the values of the MDBF norm sample. For the intervention group increases in all dimensions were documented. In the pre-post comparison the subjects showed improved mood ( $67.1 \pm 35$ to $69.5 \pm 34.3$ percentile rank); they also felt more awake $(65.1 \pm 31.5$ to $70.2 \pm 27.5$ percentile rank) and calm $(86.5 \pm 14.2$ to $89.1 \pm 11.2$ percentile rank). For the control group decreases in all subjective perceived well-being dimensions were found in the pre-post comparison (see Table 1). 
Table 1

Mean values (standard deviation) of the results of selected methods, along with statistical results ( $p$ values) for the group differences of the pre-post changes

\begin{tabular}{|c|c|c|c|c|c|}
\hline \multirow[t]{2}{*}{ Test } & \multicolumn{2}{|c|}{ Intervention group } & \multicolumn{2}{|c|}{ Control group } & \multirow{2}{*}{$\begin{array}{l}p \text { Value (group } \\
\text { differences) }\end{array}$} \\
\hline & pre & post & pre & post & \\
\hline \multicolumn{6}{|l|}{ General examination } \\
\hline $\operatorname{HR}_{\text {Rest }}\left(\min ^{-1}\right)$ & $74(13)$ & $68(11)$ & $69(10)$ & $73(14)$ & 0.058 \\
\hline BMI $\left(\mathrm{kg} / \mathrm{m}^{2}\right)$ & $26.3(3.2)$ & $25.6(3.1)$ & $26.0(3.2)$ & $26.2(2.9)$ & 0.014 \\
\hline \multicolumn{6}{|l|}{$M D B F$} \\
\hline Good - bad (percentile rank) & $67.1(35.0)$ & $69.5(34.4)$ & $73.7(24.1)$ & $57.6(35.9)$ & 0.025 \\
\hline Awake - tired (percentile rank) & $65.1(31.5)$ & $70.2(27.5)$ & $61.1(38.7)$ & $34.0(29.3)$ & 0.008 \\
\hline Calm - nervous (percentile rank) & $86.5(14.2)$ & $89.1(11.2)$ & $87.3(8.1)$ & $66.6(30.6)$ & 0.021 \\
\hline \multicolumn{6}{|l|}{ Maximum strength } \\
\hline Trunk flexion ( $\%$ from reference) & $127.8(18.3)$ & $124.7(17.4)$ & $128.8(28.0)$ & $124.8(35.8)$ & 0.721 \\
\hline Trunk extension ( $\%$ from reference) & $128.8(15.7)$ & $130.3(19.9)$ & $137.5(24.4)$ & $124.5(30.8)$ & 0.086 \\
\hline \multicolumn{6}{|l|}{ Strength endurance } \\
\hline Back muscles (s) & $114.1(67.4)$ & $160.8(81.9)$ & $98.3(46.2)$ & $108.5(49.0)$ & 0.086 \\
\hline Abdominal muscles (s) & 44.7 (31.6) & $82.2(55.8)$ & $80.0(67.6)$ & $67.6(69.6)$ & 0.057 \\
\hline Shoulder muscles (s) & $102.3(44.4)$ & $104.9(38.8)$ & $112.8(33.0)$ & $91.2(27.4)$ & 0.077 \\
\hline
\end{tabular}

The medical check-up revealed higher motion flexibility of some joints (e.g. knee extension) in the pre-post comparison. Altogether, only few effects in joint flexibility and differences of the various functionality tests could be found (results not shown).

The results of the maximum strength test of the trunk are shown in Table 1. An increase in maximum strength could only be found for the trunk extension in the intervention group (128.8 \pm 15.7 to 130.3 \pm 19.9 mean percentage from reference). The group comparisons of the pre-post differences lead to no statistically significant effects.

The strength endurance tests showed increases for all investigated muscles for the intervention group, whereas for the control group only slight increases for the strength endurance of the back muscles were found in the pre-post comparison (see Table 1). The group differences were not statistically significant.

\section{Discussion and conclusions}

According to the analyses of the activity logs and CUELA measurements, the intervention group stood significantly longer and sat less during VDU work. This different behavior is referable to the use of the sit-stand tables in the intervention group which was not available for the control group. Regarding the body dynamics, the intervention group showed also significant increases in physical activity compared to the control group. These were reflected by higher step counts measured with AiperMotion and elevated
PAI levels of all body areas in the pre-post comparison of CUELA measurements. In addition, the CUELA pre-post comparison revealed a slight increase of the percentage of walking in the intervention group. This increase in body dynamics is probably caused by the preventive measures aimed at the behavior. However, the changes in body dynamics were not strong enough to produce a measurable change in energy expenditure. This might be due to the fact that the intervention subjects primarily used the breaks between the VDU work to perform additional PA. Since break times are limited the absence of significant group difference in energy expenditure can thus be explained by the lack of opportunities to perform PA at VDU work-places. It is therefore important, to develop and implement further PA enhancing measures, which lead to a substantial increase in energy expenditure and physical activity at VDU workplaces.

The intervention group also showed improvements of the perceived well-being. The subjects stated better mood and felt more awake and calm. The MDBF questionnaire turned out to be an applicable instrument in an assessment inventory for PA.

The medical check-up revealed only slight changes and a few significant results. This is most likely due to a ceiling effect: Since this was a healthy sample, the ranges of motion of both groups were already within the normal range before the intervention started. Since the check-up was originally designed to diagnose deficits, improvements could only be detected if the initial values were below the norm. Therefore, the applied medical check-up has only 
limited suitability for effect analysis of PA enhancing measures in a healthy collective.

The assessment of maximum strength and strength endurance showed similar effects. As expected, the applied measures led to few significant increases of the maximum strength and strength endurance.

Overall, the preliminary results of the pilot study suggest that the intervention was effective and several tested methods were suitable to quantify PA intervention effects at VDU workplaces. The lack of significant results with some methods can imply either that the method was not appropriate (e.g. due to small sample size or ceiling effects) or the intervention was not sensitive for its specific methodology. Altogether, a selectively shortened method inventory that consists of a combination of standardized questionnaires to assess well-being and musculoskeletal disorders (results not shown here) together with activity measurement systems and medical-check-ups seems promising for future analyses of PA intervention effects at VDU workplaces.

\section{Acknowledgements}

This project was initiated and funded by the German Social Accident Insurance (DGUV).

\section{References}

[1] Aarås, A.; Horgen, G.; Ro, O.: Work with visual display unit: health consequences. International J. Human-Computer Interaction 12 (2000), 107-134.
[2] Baumann, A.; Phongsavan, P.; Schöppe, S.; Owen, N.: Physical activity measurement - a primer of health promotion. Promot. Educ. 2006; 13:92-103.

[3] Dishman, R. K.; Oldenburg, B.; O'Neal, H.; Shepard, R. J.: Workplace physical activity interventions. Am. J. Prev. Med. 15 (1998), 344-361.

[4] Dugdill, L.; Brettle, A.; Hulme, C.; McCluskey, S.; Long, A. F.: Workplace physical activity interventions: a systematic review. Int. J. Workplace Health Management. 1 (2008), 20-40.

[5] Oesch, P.; Hilfiker, R.; Keller, S.; Kool, J.; Schädler, S.; TalAkabi, A.; Verra, M.; Widmer-Leu C.: Assessments in der muskuloskelettalen Rehabilitation. Huber, Bern 2007

[6] Parent-Thirion, A.; Fernández, M.E.; Hurley, J.; Vermeylen, G.: Fourth European Working Conditions Survey. European Foundation for the Improvement of Living and Working Conditions, Dublin 2007

[7] Pate, R.R.; Pratt, M.; Blair, S.N.; Haskell, W.L.; Macera, C.A.; Bouchard, C. et al.: Physical activity and health. A recommendation from the Centers for Disease Control and Prevention and the American College for Sports Medicine. JAMA (1995), 273, 402-7.

[8] Proper, K. I.; Koning, M.; van der Beek, A. J.; Hildebrandt, V. H.; Bosscher, R. J.; van Mechelen, W.: The Effectiveness of Worksite Physical Activity Programs on Physical Activity, Physical Fitness and Health. Clin. J. Sport Med. 13 (2003), 106-117.

[9] Spallek, M.; Kuhn, W.; Schwarze, S.; Hartmann, B.: Arbeitsmedizinische Vorsorge bei Belastungen des Muskel-SkelettSystems - Teil 2: Funktionsorientierte Untersuchungssystematik (fokus ${ }^{\circledR}$ ) des Bewegungsapparates in der Arbeitsmedizin. Arbeitsmed. Sozialmed. Umweltmed. 40 (2005), 244-250.

[10] Steyer, R.; Schwenkmezger, P.; Notz, P. and Eid, M.: Der Mehrdimensionale Befindlichkeitsfragebogen (MDBF). Hogrefe, Göttingen 1997

[11]Weber, B.; Hermanns, I.; Ellegast, R.; Kleinert, J.: A personcentered measurement system for quantification of physical activity and energy expenditure at workplaces. In: Karsh B-T, editor. Ergonomics and Health Aspects, HCII 2009. Berlin: Springer; LNCS 5624, 121-130.

[12] Weber, B.: Entwicklung und Evaluation eines Bewegungsmesssystems zur Analyse der physischen Aktivität. IFAReport 2/2011. Hrsg.: Deutsche Gesetzliche Unfallversicherung (DGUV), Sankt Augustin 2011 